\title{
Propuesta para la aplicación de métodos ágiles en el diseño conceptual de un dron
}

| Fecha de recibido: 31 de julio del 2020 | Fecha de aprobación: 25 de septiembre del 2020 |

\section{Germán Barragán}

Magíster en Transporte aéreo y Aeropuertos

Universidad Pontificia Bolivariana

Grupo de Investigación en ingeniería Aeroespacial

Rol de investigador: teórico, experimental y escritura https://orcid.org/0000-0002-0381-8671

$\bowtie$ german.barragan@upb.edu.co

\section{Germán Urrea}

Magíster en Administración

Universidad Pontificia Bolivariana Grupo de Investigación en ingeniería Aeroespacial

Rol de investigador: teórico y escritura https://orcid.org/0000-0001-7245-005X

$\triangle$ german.urrea@upb.edu.co
Juliana Andrea Niño Navia

Magíster en Ingeniería Aeronáutica y Mecánica

Universidad Pontificia Bolivariana

Grupo de Investigación en ingeniería Aeroespacial

Rol de investigador: escritura

https://orcid.org/0000-0003-0465-0409

$\triangle$ juliana.nino@upb.edu.co

Cómo citar este artículo: Barragán, G., Urrea, G., \& Niño Navia, J. A. (2020). Propuesta para la aplicación de métodos ágiles en el diseño conceptual de un dron. Revista Ciencia y Poder Aéreo, 15(2), 110-121. https://doi.org/10.18667/cienciaypoderaereo.671 


\section{Propuesta para la aplicación de métodos ágiles en el diseño conceptual de un dron}

\section{Proposal for the Use of Agile \\ Methods in the Conceptual Design of a Drone}

Resumen: cuando se desarrolla un nuevo producto aeroespacial, es esencial evaluar su rendimiento. Los drones se consideran un producto noble con un enorme potencial de impacto en la industria de la aviación en los próximos años. El diseño de un dron certificado es una actividad compleja en la que interactúan varias áreas del conocimiento. Normalmente se plantea en fases que abarcan desde la viabilidad hasta la producción. En este artículo se propone el uso de metodologías ágiles para el diseño conceptual de un dron. Diversos estudios han demostrado el potencial para abordar desafíos similares mediante el uso de métodos análogos a aquellos que fueron empleados en el desarrollo de software (como las metodologías ágiles). El Scrum fue el método ágil seleccionado para su adaptación al diseño del dron con el fin de gestionar mejor la complejidad del proceso de diseño (incluyendo las alteraciones de este). Finalmente, se presenta una propuesta para la aplicación de este método mostrando las diferentes etapas diseño y sus beneficios potenciales.

Palabras clave: desarrollo de producto; diseño conceptual; dron; planificación de diseño; Scrum.

Abstract: Assessing performance is essential when a new aerospace product is being developed. Drones are considered a noble product with enormous potential for the aviation industry in the upcoming years. In this context, certified drone designing is a complex activity that requires the interaction of various areas of knowledge and that is normally approached in phases ranging from feasibility to production. Therefore, this article proposes the use of agile methodologies for the conceptual design of a drone, considering that several studies have shown the potential of this type of methods for addressing similar challenges, as in the case of software development. Scrum was the agile framework selected for the design of the device and thus better manage product complexity and process disturbances. A proposal for the application of this method, showing the different design stages, is presented in the final section of the paper.

Keywords: Product development; conceptual design; drone; design planning; scrum.

Resumo: Quando um novo produto aeroespacial é desenvolvido, é essencial avaliar o desempenho. Os drones são considerados um produto nobre com enorme potencial de impacto na indústria da aviação nos próximos anos. O projeto de um drone certificado é uma atividade complexa, que requer a interação de várias áreas do conhecimento e é normalmente considerada em fases que variam da viabilidade à produção. Este artigo propõe o uso de metodologias ágeis para o projeto de design conceitual de um drone, dado que diversos estudos mostraram o potencial de enfrentar desafios similares usando métodos semelhantes ou análogos usados no desenvolvimento de software, como metodologias ágeis. O Scrum foi o método ágil selecionado para ser adaptado ao design do drone, a fim de gerenciar melhor a complexidade do produto e as alterações do processo. Por último, é apresentada uma proposta para a aplicação desse método, mostrando as diferentes etapas do projeto.

Palavras-chave: Desenvolvimento de produto; Projeto conceitual; Drone; Planejamento de projeto; Scrum. 


\section{Introducción}

El lanzamiento de nuevas tecnologías o productos eficientes y acordes con los requisitos del mercado es uno de los factores de éxito para las empresas en un ambiente competitivo en el que predomina la globalización y la competencia de precios (McDermott \& O'Connor, 2002). El cambio en las preferencias del mercado, los requisitos de flexibilidad y el acortamiento de los ciclos de vida del producto exigen que las empresas desarrollen bienes con características mejoradas y con un diseño y operación innovadores (Schuh, 2012). La industria de la aviación no es ajena a estas exigencias. A diario se ve en la obligación de desarrollar conceptos nuevos y revolucionarios para sus productos.

El interés en los drones o vehículos aéreos no tripulados (UAV) ha crecido sostenidamente (US Deparment of Defense, 2007; Luppicini \& So, 2016) y se pronostica un aumento significativo de su uso durante los próximos años (Lee et al., 2018). Esta previsión del aumento de la demanda trae como resultado la necesidad de crear nuevos diseños de vehículos con diversas capacidades para aplicaciones militares y civiles. Esta necesidad se ha expandido incluso hacia el desarrollo de drones con la capacidad de operar autónomamente en diferentes entornos y de realizar diversas misiones en todo el territorio mundial (Hassanalian \& Abdelkefi, 2017). Pueden ser empleados en tareas de búsqueda y rescate, protección del medio ambiente, transporte, vigilancia y monitoreo, fotografía, inspección, meteorología, mapeo, entretenimiento, entre muchas otras.

Los drones pueden ser clasificados de diversas maneras. Comúnmente se clasifican de acuerdo con la plataforma de aplicación, sea esta civil o militar (Lavallée, 2019); por el tamaño y peso (Hassanalian \& Abdelkefi, 2017), encontramos desde nano drones hasta drones pesados, y de acuerdo al grado de autonomía (Albeaino et al., 2019). En esta última categoría encontramos principalmente tres tipos. Primero, sistemas completamente autónomos, sin ninguna intervención humana durante su operación. Estos sistemas se valen de sistemas de navegación y un software especializado que permite predeterminar las rutas y las tareas que se deben llevar a cabo. Segundo, sistemas semiautónomos, que permiten una interacción entre los sistemas autónomos y el hombre. Finalmente, los sistemas manuales, que son controlados por un piloto desde tierra.

Es importante aclarar los conceptos relacionados con la nomenclatura utilizada en este tipo de vehículos aéreos. En principio, se puede establecer una diferencia entre el sistema y el vehículo. Los términos UAS (Unmanned Aircraft Systems), RPAS (Remotely Piloted Aircraft System) y S-ART (Sistema de aeronave remotamente tripulada) son utilizados para referirse al vehículo y al conjunto de elementos necesarios para su mando y control. Entre estos elementos se incluyen la estación de mando y control, los enlaces para la comunicación entre la estación y el vehículo y cualquier otro componente requerido para la operación. Por su parte, términos tales como, UAV (Unmanned Aerial Vehicle), RPA (Remotely Piloted Aircraft) y ART (Aeronave Remotamente Tripulada) hacen referencia exclusivamente al vehículo; es decir, al aparato que se encuentra en vuelo. Por último, el término "drone" o "dron" es una expresión genérica que se utiliza para referirse, indiferentemente, a cualquier aeronave no tripulada o remotamente piloteada. El presente documento habla solamente del diseño del vehículo y no del sistema, por lo que se empleará el termino de uso genérico dron.

En general, el proyecto de desarrollar nuevas máquinas voladoras es arriesgado y extremadamente complejo; es una empresa desafiante de ingeniería (Komarov, et al., 2011) que requiere la integración de múltiples sistemas. La creciente utilización de elementos mecatrónicos en forma de componentes aerodinámicos, mecánicos, electrónicos y de software aumenta la complejidad del proyecto durante el desarrollo. Por esto, los fabricantes buscan controlar cada vez más esta complejidad adoptando un enfoque revolucionario.

Los métodos de desarrollo ágil ya han demostrado su capacidad de optimizar los principales factores de éxito de un proyecto (Schuh et al., 2018). Es posible encontrar en la literatura investigaciones centradas en mejorar los resultados en el trabajo, en términos de eficiencia y eficacia, con métodos ágiles 
usados en proyectos complejos y en el desarrollo de productos. De esta literatura se destacan Ghimire et al. (2020), Salvato y Luplume (2020), Huikkola y Kohtamäki (2020), Borda et al., 2019), Ciric et al. (2018). Estos estudios han demostrado el potencial de la adopción de los métodos agiles para enfrentar los desafíos en el desarrollo de sistemas y elementos mecánicos altamente complejos. En la década de 1990, el desarrollo de software se enfrentó a situaciones de mercado y desafíos similares a los que se esperan para los procesos de diseño o fabricación de drones.

Este documento propone la aplicación de los principios de métodos ágiles para los proyectos de diseño conceptual de drones. Estos principios permiten manejar mejor la complejidad del producto y enfrentar las posibles alteraciones requeridas durante el proceso de diseño, e incluso las derivadas de la operación de este.

\section{Proyecto de diseño de un dron}

El proyecto de desarrollo de un dron tiene componentes análogos a los del diseño de una aeronave tradicional. Si se considera el ciclo de desarrollo estructurado que forma la base de los programas comerciales de Airbus, se encuentra que todo el proceso está dividido en cinco fases principales: viabilidad, concepto, definición, desarrollo y serie. Se requieren años de evolución para pasar de la idea de producto establecido inicialmente al desarrollo final de la aeronave (Pardessus, 2004).

Muchos métodos de diseño de aeronaves como los descritos (Anderson, 1999; Raymer, 2012; Roskam, 2004) también incluyen los aspectos del diseño de drones y son una combinación de métodos de dimensionamiento analítico y semi-empírico. Estos pueden ser utilizados para llevar a cabo un estudio de diseño completo y un análisis aerodinámico que han sido validados con aplicaciones en aviones comerciales y drones (Panagiotou et al., 2016).

Generalmente, durante el diseño y desarrollo de una aeronave, se utilizan adaptaciones de estos métodos u otros durante el proceso. Como ejemplo de estos casos, en la figura 1 se presenta una hoja de ruta de la herramienta de diseño conceptual propuesta por Panagiotou, Fotiadis-Karras y Yakinthos (2018).

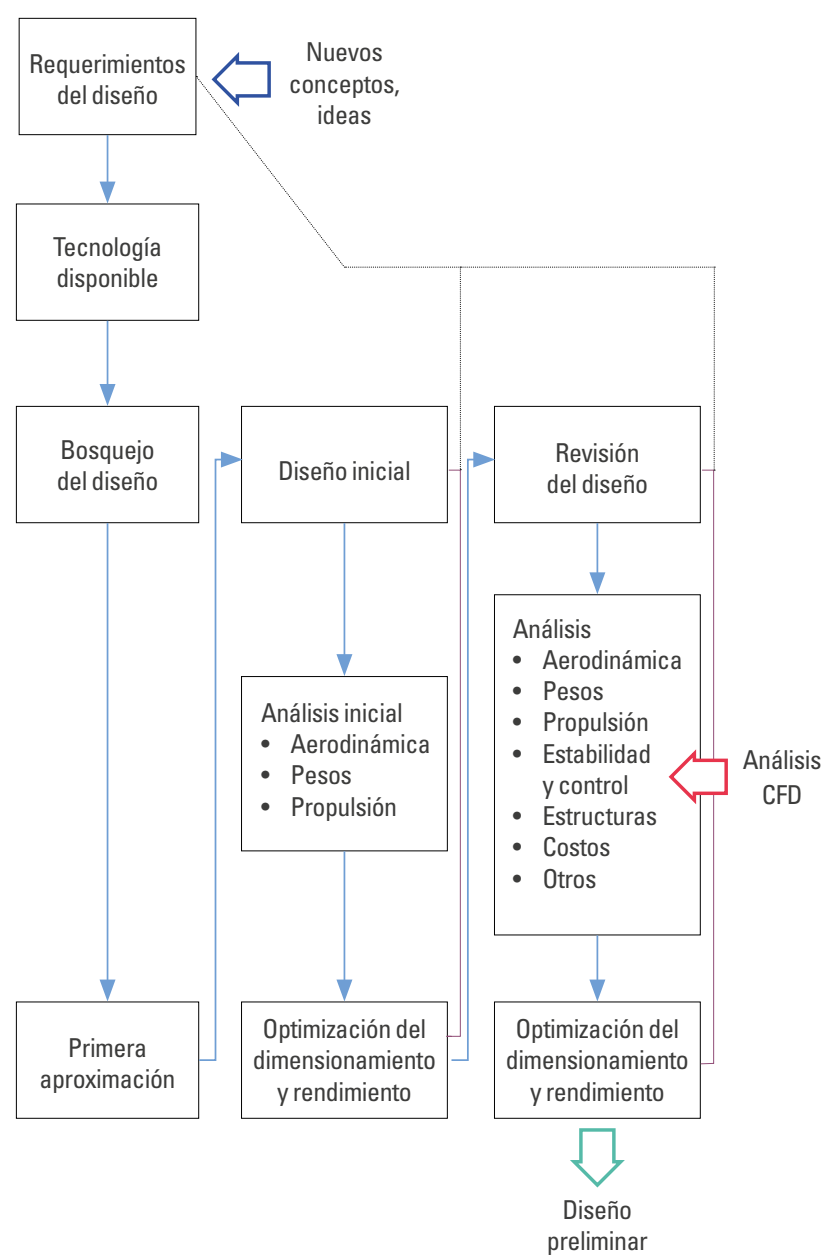

Figura 1. Hoja de ruta de para el diseño conceptual de una aeronave Fuente: Panagiotou et al. (2018).

En el caso específico del diseño de una aeronave no tripulada, el proceso se da a través de un ciclo de vida de diseño. Este ciclo comienza con el desarrollo de un concepto: una idea general del vehículo. El concepto define formas, medios y parámetros para proporcionar una alta eficiencia y competitividad al aparato. Con esto se busca la superioridad en comparación con otros de su tipo que ya se encuentren en operación o 
que hayan sido diseñados. En esta parte es muy importante considerar la tecnología disponible, ya que esta rige en gran medida las pautas de diseño.

El concepto de un futuro dron está determinado por los requisitos para las características funcionales y de rendimiento correspondientes, definidas por un cliente. Algunas de las funciones que pueden ser requeridas son: inspección industrial, departamento de bomberos, teledetección, búsqueda y rescate, fotografía aérea, mensajería y una vasta gama de aplicaciones personalizadas. Estas aplicaciones tienen una serie de diversos requisitos de naturaleza jerárquica. Los requisitos de nivel superior se descomponen en niveles inferiores hasta que alcanzan un nivel de detalle que los responsables de implementar el proyecto pueden usar para las fases de fabricación, construcción y ensamble. La figura 2 muestra diferentes configuraciones de drones de acuerdo con sus aplicaciones y usos.
Básicamente, la tarea de diseño implica la manipulación de parámetros físicos para alterar la aerodinámica, la estructura, la propulsión y los sistemas. A su vez, esto influye directamente sobre el rendimiento del vehículo y, por lo tanto, sus capacidades de misión. Los primeros cálculos de dimensionamiento se basan principalmente en relaciones semi-empíricas y datos estadísticos, así como en algunos bocetos de conceptos iniciales. El siguiente paso es estimar algunos parámetros clave de rendimiento y elementos de diseño, como las especificaciones de superficie aerodinámica, el área del ala y las especificaciones de propulsión. Estos parámetros sirven como la base sobre la cual se dibujan los diseños/candidatos de configuración detallados (Panagiotou et al., 2018). Las etapas de diseño son interactivas y de naturaleza altamente iterativa.

Las diferentes tareas de la fase de diseño conceptual requieren un gran esfuerzo de los equipos de
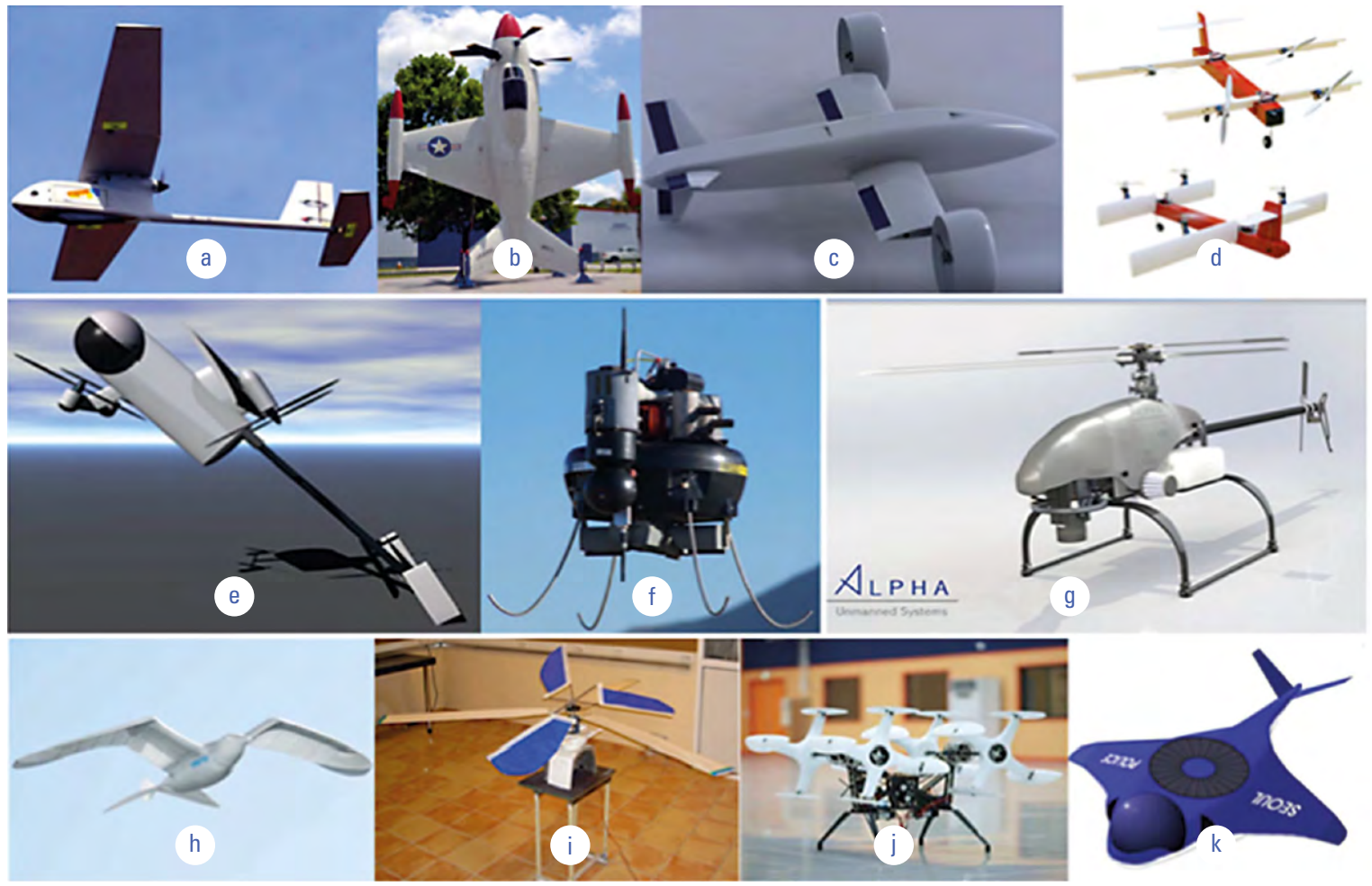

Figura 2. Diferentes configuraciones de drones. (a) Tradicional con aterrizaje y despegue horizontal; (b) aterrizaje y despegue vertical; (c) rotor inclinable; (d) ala inclinable; (e) cuerpo inclinable; (f) ducted fan; (g) helicóptero; (h) alas de gaviota; (i) ornitóptero; (j) multirrotor, y (k) geometría no convencional. Fuente: Hassanalian \& Abdelkefi (2017) 
diseño. Por ello es necesario un análisis que, a futuro, permita obtener mejoras en los procesos, en la forma en que los equipos de diseño cooperan, la forma en que intercambian la información, la manera de estimar el esfuerzo de desarrollo del proyecto y cómo se manejan los cambios de diseño. En muchos casos, el valor del gerente de proyecto se cuantifica en términos de poder analizar estos problemas sistemáticamente y responder a las situaciones rápidamente.

Después de que el equipo del proyecto concluye el diseño, comienza la etapa de fabricación. Sin embargo, durante esta es posible que se necesiten algunos ajustes del diseño.

\section{Peculiaridades del desarrollo de un dron}

Para comprender mejor la complejidad de la actividad, y la necesidad de introducir nuevas formas de controlarla para maximizar su calidad, es necesaria una breve descripción de las peculiaridades de los proyectos de desarrollo de dron. Primero, la industria de la aviación se considera un negocio conservador (Esposito \& Raffa, 2004): funciona con una pequeña cantidad de unidades vendidas, en algunos casos una única unidad. Las economías de escala tienen un efecto menor y los costos de desarrollo son una porción significativa del precio. Es muy común que las instalaciones de producción estén dedicadas a un tipo de vehículo específico. Los drones se consideran un producto complejo y requieren una gran cantidad de conocimiento y calidad organizacional. Un proceso de certificación es un requisito especial e influye en el desarrollo de nuevos productos, ya que los desarrolladores tienden a favorecer los conceptos evolutivos sobre los conceptos revolucionarios (Glas \& Ziemer, 2009; Hodgkinson \& Johnston, 2018).

La complejidad del diseño de un dron se debe fundamentalmente a la cantidad de requerimientos que debe cumplir. Estos van desde las posibles aplicaciones y rendimiento de este hasta las regulaciones existentes. Teniendo en cuenta estos requerimientos, se pueden identificar dos factores principales que afectan el diseño: factores tecnológicos y operacionales. Dentro de los factores tecnológicos se consideran los siguientes: sensores y equipos de control; baterías y sistemas de comunicación; sistemas de navegación; capacidad de cómputo; carga útil; seguridad operacional y seguridad de los datos; factores ambientales que afecten el correcto funcionamiento de los equipos electrónicos; materiales; técnicas de manufactura, y partes móviles.

Entre los factores operacionales más importantes a considerar encontramos: condiciones ambientales; factores geográficos; sistemas de interacción y de control de acuerdo con la autonomía del dron; factores humanos; operación y mantenimiento del equipo; seguridad operacional; requerimientos de infraestructura; leyes, políticas y regulaciones, etc. (Hassanalian \& Abdelkefi, 2017).

Todos estos factores, sumados a las condiciones del mercado (alto nivel de incertidumbre, cambios constantes, necesidad de respuestas rápidas a los cambios) y a los requisitos del producto (innovación, tiempo de desarrollo corto, alta personalización, piezas hechas a pedido) hacen que cada proyecto de desarrollo tenga un alto riesgo financiero, tecnológico y de gestión.

Los métodos ágiles pueden ser explorados como una forma de abordar estos riesgos y de reducir la complejidad de los proyectos de desarrollo.

\section{Métodos ágiles}

El Manifiesto ágil se compone de cuatro valores fundamentales y doce principios de apoyo (Koch, 2004; Darrin \& Devereux, 2017). La metodología ágil aplica los cuatro valores de diferentes maneras, pero todos ellos sirven para guiar el desarrollo y la entrega de productos de alta calidad. Estos principios se muestran en la tabla 1. 
Tabla 1.

Los doce principios del manifiesto ágil

\begin{tabular}{|c|c|}
\hline \multicolumn{2}{|r|}{ Principios } \\
\hline 1 & $\begin{array}{l}\text { Complacencia del cliente mediante la entrega rápida } \\
\text { de un software útil }\end{array}$ \\
\hline 2 & $\begin{array}{l}\text { Bienvenida a los requisitos cambiantes, incluso tarde } \\
\text { o en las fases tardías del desarrollo }\end{array}$ \\
\hline 3 & Entrega de software de trabajo con frecuencia \\
\hline 4 & El software de trabajo es la principal medida de progreso \\
\hline 5 & $\begin{array}{l}\text { Desarrollo sostenible, capaz de mantener un } \\
\text { ritmo constante }\end{array}$ \\
\hline 6 & $\begin{array}{l}\text { Satisfacción del cliente mediante la entrega rápida } \\
\text { de software útil }\end{array}$ \\
\hline 7 & $\begin{array}{l}\text { Cooperación cercana y diaria entre empresarios } \\
\text { y desarrolladores }\end{array}$ \\
\hline 8 & $\begin{array}{l}\text { La ubicación conjunta, la conversación directa persona } \\
\text { a persona es la mejor forma de comunicación }\end{array}$ \\
\hline 9 & $\begin{array}{l}\text { Los proyectos se basan en individuos motivados, } \\
\text { en quienes se debe confiar }\end{array}$ \\
\hline 10 & Simplicidad \\
\hline 11 & Equipos autoorganizados \\
\hline 12 & Adaptación regular a las circunstancias cambiantes \\
\hline
\end{tabular}

Fuente: adaptado de Fowler \& Highsmith (2001).

Por lo tanto, el núcleo del método ágil es que los objetivos de un proyecto se definan con menos detalles al inicio del proyecto y que se prepare un cronograma de ejecución aproximada del proyecto: el proyecto se divide en iteraciones iguales con partes asignadas del proyecto, dado por alcance del propio proyecto al ser creado. Al principio, un equipo asume las funciones más importantes y deja las menos importantes para el final. Al inicio de cada iteración se crea una especificación detallada de los productos de las iteraciones y se hace una programación precisa de las iteraciones. El plan del proyecto para la iteración es realizado por el equipo del proyecto (Stare, 2014).

Sobre su desarrollo, es posible caracterizarlo como un enfoque orientado al aprendizaje (Schuha et al., 2017). Se caracteriza por una realización temprana, incremental e iterativa de pruebas de productos y es realizado por pequeños equipos de desarrollo que reciben un alto grado de libertad creativa (Sutherland, 2013). Los equipos de desarrollo aprenden de los resultados de las pruebas y de los comentarios de los clientes e incorporan el conocimiento obtenido en los próximos ciclos de iteración.

Los métodos ágiles brindan un enfoque más "flexible" para el desarrollo de nuevos productos, adaptable a las contingencias del entorno del proyecto (Conforto et al., 2014). Algunos de estos métodos son: Scrum (Srivastava et al., 2017), desarrollo de software Lean (Kupiainen et al., 2015), Crystal (Anwer et al., 2017), desarrollo dirigido por funciones (FDD) (Nawaz et al., 2017), desarrollo adaptativo de software (Aftab, et al., 2018), método de desarrollo dinámico del sistema (DSDM) (Rohajawati et al., 2019), programación extrema (Sohaib et al., 2019).

Los métodos ágiles han recibido mucha atención. Debido a su flexibilidad y capacidad de adaptación, podrían ofrecer beneficios prometedores para las empresas manufactureras, brindar capacidad de cambio donde sea necesario y ventajas de costos al mismo tiempo. La flexibilidad introducida a través de los procesos ágiles permite un manejo eficiente de la incertidumbre del mercado y la incertidumbre técnica. En sus estudios, Takeuchi y Nonaka (1986) observaron prácticas y principios en programas de desarrollo de nuevos productos innovadores que siguieron principios ágiles. Lo mismo hicieron otros como Vinodh et al. (2010), Stare (2014), Rebentisch et al. (2018) y Mulder et al. (2014).

En el caso de la industria de la aviación, los estudios de aplicación de métodos ágiles han sido explorados, entre otros, por la Universidad Federal de Itajuba (Krupa, 2019), el Crescent Innovation \& Incubation Council (Parvez \& Toppur, 2019), la Universidad Aeronáutica Embry-Riddle (Blooshi et al., 2018), la Universidad de Cranfield (Baguley, 2018), la Universidad de Nápoles (Della Vecchia et al., 2016), la Bauhaus Luftfahrt (Glas \& Seitz, 2012) y Boeing (Ivanis, 2009).

\section{Aplicación del método ágil}

Las propiedades fundamentales de los métodos ágiles entran en conflicto con varios requisitos de un dron certificado; por ejemplo, con el requisito de llevar una 
información de seguridad rastreable. La certificación obligatoria de todos los componentes requiere una documentación detallada o el desarrollo global, que es una práctica común en la industria. En consecuencia, la industria de la aviación tradicionalmente reserva el uso de principios ágiles para la fase de diseño conceptual (Glas \& Ziemer, 2009). En esta fase, las limitaciones son menos críticas en comparación con la adaptación eficiente de herramientas y métodos, la explotación de la libertad de diseño y el manejo de conocimiento limitado. Esto hace que la aplicación de los métodos ágiles sea razonable.

Esta apuesta por la utilización de métodos ágiles fue basada su eficacia demostrada en el desarrollo de proyectos de alta complejidad en entornos altamente cambiantes. El trabajo de equipos multidisciplinares es empleado en estos entornos. Estas son situaciones bastante comunes en el proceso de diseño de un nuevo dron volador.

Entre los métodos ágiles disponibles, el Scrum fue seleccionado para adaptarse al diseño de un dron, ya que permite trabajar de forma colaborativa entre los miembros del equipo. De este modo, se pueden obtener resultados rápidos y optimizados, especialmente en entornos en los que los parámetros cambian constantemente. Utilizar la metodología Scrum en el diseño les da a los usuarios la capacidad de cambiar el tamaño, la forma y la estructura de su vehículo en función de sus necesidades específicas, como carga útil, costo, tiempo de vuelo, uso de la batería (consumo de combustible) y otros factores. El método ofrece una nueva posibilidad de saber cómo se ven y funcionan los drones.

Los tres roles principales en el marco de Scrum son: el propietario del dron (cliente), el Scrum Máster y el equipo de diseño. Juntos, estos tres roles forman el equipo Scrum (Schwaber \& Sutherland, 2016). La selección de personas para asumir estos roles debe ser la primera etapa del proyecto. El propietario del dron es responsable de transmitir una visión compartida del producto, así como su entrega a tiempo y con calidad; él se encarga de maximizar el valor del producto y el trabajo del equipo. El Scrum Máster es el responsable de que el equipo cumpla con el método Scrum y de eliminar los impedimentos de productividad para el equipo. El equipo de diseño es el grupo que realiza el trabajo y entrega un producto al final del proyecto.

Es importante tener en cuenta que un equipo de diseño involucra la interacción humana, por lo que requiere la capacidad de escuchar y responder de manera constructiva a las opiniones expresadas por otros, así como la capacidad de brindar a los demás el beneficio de la duda, brindar apoyo y reconocer los intereses y logros de los demás (Kazenbach \& Smith, 1993). Dickinson y McIntyre (1997) identificaron y definieron siete componentes centrales del trabajo en equipo. Utilizando estos componentes y sus relaciones como base, propusieron el modelo de trabajo en equipo que se aplica al equipo ágil. El modelo consiste en un ciclo de aprendizaje de los siguientes componentes básicos del trabajo en equipo: comunicación, orientación del equipo, liderazgo del equipo, monitoreo, retroalimentación, respaldo y coordinación. Teniendo esto en cuenta, es posible decir que un equipo ágil se caracteriza por estar formado por humanos, ser autogestionado, tener una buena comunicación, un gran conocimiento y experiencia, una alta capacidad para cambiar, hacer mejoras y para el trabajo iterativo. Estos factores deben tenerse en cuenta al formar el equipo de diseño.

Después de asignar los roles, se debe definir la visión del producto. Una recomendación es seguir la conclusión de Benassi, Amaral y Ferreira (2016). Para ellos, la visión del producto se identifica como el producto de una construcción que emplea varias áreas y tiene un significado asociado a la descripción del producto final. Para el caso del dron, la visión del producto puede consistir en algunas oraciones que describen una característica que un el sistema deba proporcionar. Por ejemplo, "el dron tendrá una autonomía de dos horas y una función principal de vigilancia".

Es importante recalcar que, independientemente de si el Scrum busca reducir la complejidad y la cantidad de documentos de la organización, los requisitos de trazabilidad de la aviación deben cumplirse. Para cumplir con estas dos exigencias, la recomendación es utilizar algunos documentos esenciales, incluidos el backlog del producto, el backlog de Sprint, la tabla 
Burn Down y los documentos de información del dron. En estos documentos se puede incluir una descripción del equipo, los sistemas, métodos de diseño desarrollados, cumplimiento de requisitos de certificación y otros. Además del proyecto, se pueden incluir las lecciones aprendidas, es decir, los hallazgos de las retrospectivas de Sprint, y las mejores prácticas para la futura aplicación Scrum en otros ejercicios de diseño.

El flujo de trabajo de la tarea de diseño debe ejecutarse en el marco de un proyecto de diseño grupal interdisciplinario y en pocas semanas calendario. Se caracterizan cinco flujos esenciales de tareas:

- Requisitos de diseño y regulaciones aplicadas.

- Definición de disponibilidad de tecnología.

- Proceso de diseño de drones.

- Dimensionamiento, configuración y optimización del rendimiento.

- Modelado, prueba y simulación.

Los principios de Scrum se utilizaron para resolver problemas relacionados con la naturaleza altamente iterativa e interrelacionada de las tareas simultáneas. La figura 3 ilustra la división del desarrollo de drones en varios sprints.
El Sprint es el elemento vital del Scrum. Se trata de un periodo de tiempo de un mes o menos, durante el cual se crea una mejora incremental en el producto "listo", que se considera utilizable y potencialmente liberable. Para esto, se deben crear sprints con duraciones consistentes a lo largo del esfuerzo de desarrollo. La idea es establcer sprints limitados a un máximo de un mes de duración. Las tareas para realizar durante el sprint se plantean dentro de la planificación del Sprint. Este plan es creado por el trabajo colaborativo de todo el equipo Scrum. La figura 4 muestra un detalle del sprint de diseño propuesto.

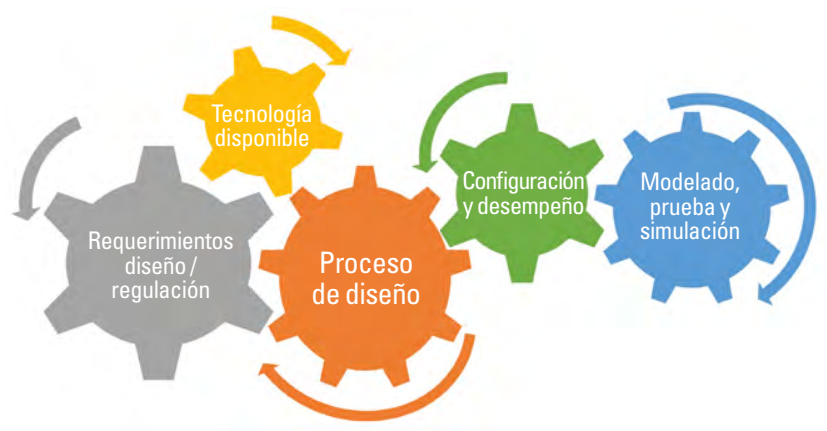

Figura 4. Sprint de diseño detallado Fuente: elaboración propia.

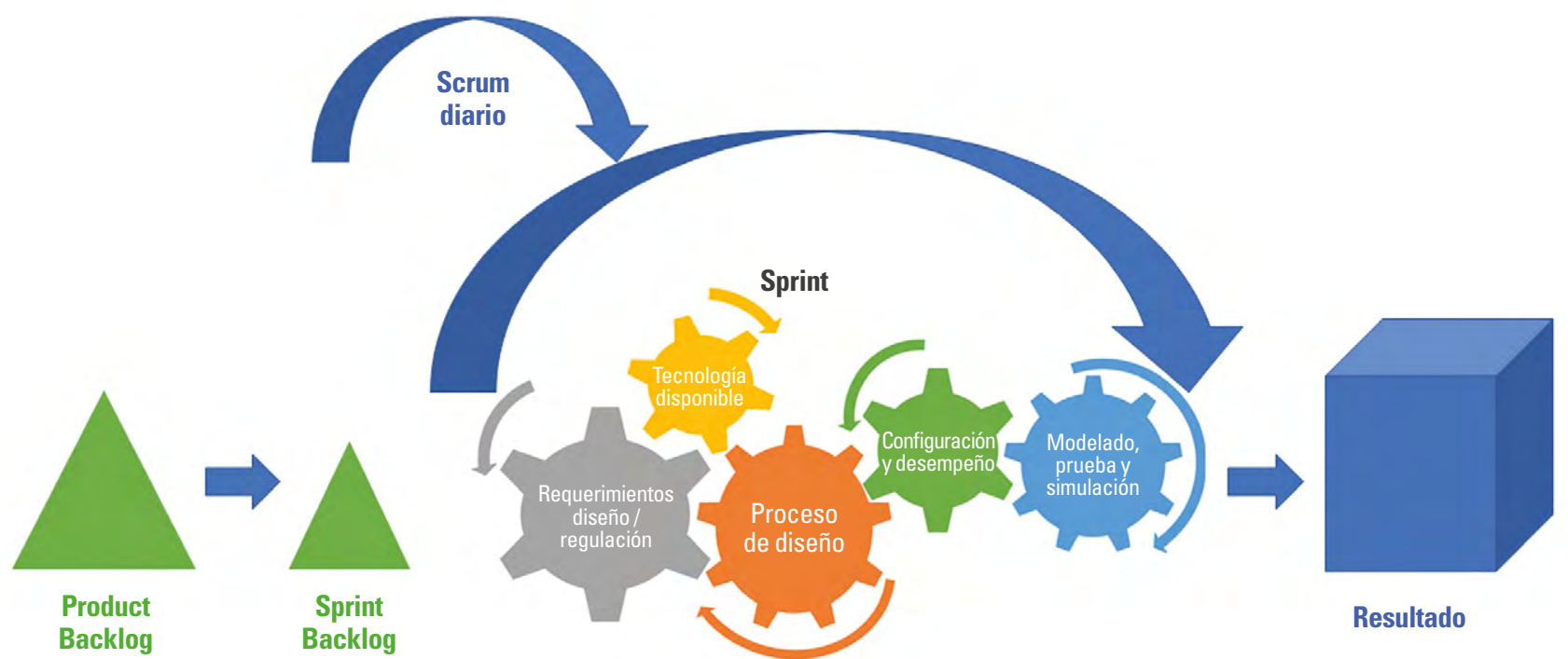

Figura 3. Proceso de diseño del dron Fuente: elaboración propia. 
Los sprints deben planificarse de acuerdo con los backlogs de productos predefinidos, llevarse a cabo y revisarse para cumplir con los cronogramas del plan general del proyecto. Esto asegura interfaces bien definidas para tareas posteriores dependientes. Los alcances detallados de trabajo para los sprints fueron negociados entre los equipos y los clientes correspondientes durante las reuniones. La reunión del proyecto representó una herramienta importante para obtener la información, realizar los cambios necesarios y optimizar los resultados producidos durante el proyecto.

\section{Conclusiones}

Las metodologías y técnicas ágiles tienen un enorme potencial para facilitar las técnicas de operación y generación de nuevos productos dentro de las empresas aeroespaciales; permiten optimizar las operaciones de negocio en términos de eficiencia, tiempo de comercialización y calidad.

El proceso de fabricación de un producto aeroespacial es muy complejo. Es necesario crear un plan de gestión de proyectos que sea guiado por la idea de proporcionar ese producto al usuario final e incluya el mantenimiento del producto durante el tiempo que el usuario lo use. Por estas razones, la aplicación de la metodología ágil se extendió al proceso de desarrollo completo. Esta tarea requiere que los métodos ágiles se adapten a los proyectos de desarrollo a gran escala manteniendo sus beneficios y estándares de calidad.

La aplicación propuesta de principios ágiles a un proyecto de diseño de drones mejora la gestión de la complejidad durante el proceso y ayuda a encontrar soluciones de mayor eficiencia para sus desafíos o problemas. La selección de la metodología Scrum es la más apropiada para el caso específico del proyecto de diseño.

Como se mostró en muchos casos, un factor clave para la implementación exitosa de las metodologías ágiles en una empresa es la capacidad de analizar problemas sistemáticamente, responder a situaciones rápidamente, aceptar cambios y manejar los nuevos desafíos que tienen los miembros del equipo y los ejecutivos. Los resultados del proyecto dependen significativamente del desempeño del equipo.

\section{Referencias bibliográficas}

Aftab, S., Nawaz, Z., Anwar, M., Anwer, F., Bashir, M., \& Ahmad, M. (2018). Comparative Analysis of FDD and SFDD. International Journal of Computer Science and Network Security, 18(1), 63-70. http://paper.ijcsns.org/07_book/201801/20 180108.pdf

Albeaino, G., Masoud, G., \& Franz, B. (2019). A systematic rewiev of unmmaned aerial vehicle application areas and thecnologies in the aec domain. Journal of information technology in construction, 24. https://itcon.org/paper s/2019_20-ITcon-Albeaino.pdf

Anderson, J. D. (1999). Aircraft Performance and Design. WCB/ McGraw-Hill.

Anwer, F., Aftab, S., Waheed, U., \& Muhammad, S. (2017). Agile Software Development Models TDD, FDD, DSDM, and Crystal Methods: A Survey. International Journal of Multidisciplinary Sciences and Engineering, 8(2), 1-10. https:// www.researchgate.net/publication/316273992_Ag ile_Software_Development_Models_TDD_FDD_DSDM_ and_Crystal_Methods_A_Survey

Baguley, P. (2018). Agile development and Cost of UAV.

Benassi, J. L., Amaral, D. C., \& Ferreira, L. (2016). Towards a conceptual framework. Journal of Operations \& Production Management, 36(2), 200-219. https://doi.org/10.1108/ IJOPM-11-2013-0511

Blooshi, M. A., Jafer, S., \& Patel, K. (2018). Review of Formal Agile Methods as Cost-Effective Airworthiness Certification Processes. Journal of Aerospace Information Systems, 15(7), 1-14. https://doi.org/10.2514/1.1010601

Borda, J., Trabasso, L., \& Pessoa, M. (2019). Agile Management in Product Development. Research-Technology Management, 62(5), 63-67. https://doi.org/10.1080/08956308.20 19.1638488

Ciric, D., Bojan, L., Gracanin, D., Palcic, I., \& Zivlak, N. (2018). Agile Project Management in New Product Development and Innovation Processes: Challenges and Benefits Beyond Software Domain. Conference: 2018 IEEE International Symposium on Innovation and Entrepreneurship (TEMS-ISIE), 1-19. https://doi.org/10.1109/TEMS-ISIE. 2018.8478461 
Conforto, E., Salum, F., Amaral, D., Silva, S., \& Almeida, L. (2014). Can Agile Project Management Be Adopted by Industries Other than Software Development? Project Management Journal, 45(3), 21-34. https://doi.org/10.10 02/pmj.21410

Darrin, M., \& Devereux, W. (2017). The Agile Manifesto, design thinking and systems engineering. Annual IEEE International Systems Conference (SysCon), 1-5. https://doi. org/10.1109/SYSCON.2017.7934765

Della Vecchia, P., De Marco, A., Stingo, L., \& Nardone, G. (2016). The Agile Method Applied to Aircraft Design at Univeristy of Naple. 6th EASN International Conference On Innovation in European Aeronautics Research.

Dickison, T., \& McIntyre, R. M. (1997). A conceptual framework of teamwork measurement. En Brannick, M, Salas, T., Team Performance Assessment and Measurement: Theory, Methods, and Applications (pp. 19-43). Psychology Press.

Esposito, E., \& Raffa, L. (2004). Evolution of the supply chain. 15th International Annual IPSERA.

Fowler, M., \& Highsmith, J. (2001). The agile manifesto. Software Development.

Ghimire, D., Charters, S., \& Gibbs, S. (2020). Scaling Agile Software Development Approach in Government Organization in New Zealand. ICSIM '20: Proceedings of the 3rd International Conference on Software Engineering and Information Management, 100-104. https://doi.org/10.1 145/3378936.3378945

Glas, M., \& Seitz, A. (2012). Application of Agile Methods in Conceptual Aircraft Design. Deutsche Gesellschaft für Luftund Raumfahrt-Lilienthal-Oberth eV. https://www.dglr. de/publikationen/2012/281384.pdf

Glas, M., \& Ziemer, S. (2009). Challenges for Agile Development of Large Systems in the Aviation Industry. Proceedings of the 24th ACM SIGPLAN Conference companion on Object oriented programing systems languages and aplications, 901-908.

Hassanalian, M., \& Abdelkefi, A. (2017). Classifications, Applications, and Design Challenges of Drones: A Review. Progress in Aerospace Sciences, 91, 99-131. https://doi. org/10.1016/j.paerosci.2017.04.003

Hodgkinson, D., \& Johnston, R. (2018). Aviation Law and Drones: Unmanned Aircraft and the Future of Aviation. Routledge.

Huikkola, T., \& Kohtamäki, M. (2020). Agile new solution development in manufacturing companies. Technology
Innovation Management Review, 10(3), 16-23. https://tim review.ca/article/1333

Ivanis, D. (2009). Where Lean and Technology Meet. Boeing. com. https://www.boeing.com/news/frontiers/archi ve/2009/.../i_ca01.pdf

Kazenbach, J. R., \& Smith, D. K. (1993). The discipline of teams. Harvard Business Review, 83(7), 111-120.

Koch, S. (2004). Agile principles and open source software development: A theoretical and empirical discussion. Lecture notes in computer science, 85-93.

Komarov, V. A., Borgest, N. M., Vislov, I. P., Vlasov, N. V., Kozlov, D. M., Korolkov, O. N., \& Maynskov, V. N. (2011). Conceptual Aircraft Design. Samara State Aerospace University.

Krupa, G. P. (2019). Application of Agile Model-Based Systems Engineering in aircraft conceptual design. Aeronautical Journal, 123(1268), 1561-1601. https://doi.org/10.1017/ aer.2019.53

Kupiainen, E., Mäntylä, M., \& Itkonen, J. (2015). Using metrics in Agile and Lean Software Development-A systematic literature review of industrial studies. Information and Software Technology, 62, 143 -163. https://doi.org/10.10 16/j.infsof.2015.02.005

Lavallée, C. (2019). The EU Policy for Civil Drones: The Challenge of Governing Emerging Technologies. Institute for European Studies Policy Brief. https://core.ac.uk/download/ pdf/200760322.pdf

Lee, J., Tan, H., Crandall, D., \& Šabanović, S. (2018). Forecasting Hand Gestures for Human-Drone Interaction. HRI'18: COMpanion of the 2018 ACM/IEEE International Conference on Human-Robot Interaction, 167-168. https://doi. org/10.1145/3173386.3176967

Luppicini, R., \& So, A. (2016). A Technoethical Review of Commercial Drone Use in the Context of Governance, Ethics, and Privacy. Technology in Society, 46, 109-119. https:// doi.org/10.1016/j.techsoc.2016.03.003

McDermott, C., \& O'Connor, G. (2003). Managing radical innovation: an overview of emergent strategy issues. Journal of Product Innovation Management, 19(6), 424-438. https://doi.org/10.1111/1540-5885.1960424

Mulder, F., Verlinden, J., \& Maruyama, T. (2014). Adapting Scrum Development Method for Development of Cyber-Phisical Systems. Proceedings of TMCE 2014, 19-23. https://www.researchgate.net/publication/262419522

Nawaz, Z., Aftab, S., \& Anwer, F. (2017). Simplified FDd Process Model. Modern Education and Computer Science, 9(9), 53-59. https://doi.org/10.5815/ijmecs.2017.09.06 
Panagiotou, P., Fotiadis-Karras, S., \& Yakinthos, K. (2018). Conceptual Design of a Blended Wing Body MALE UAv. Aerospace, Science and Technology, 73, 32-47. https://doi. org/10.1016/j.ast.2017.11.032

Panagiotou, P., Kaparos, P., Salpingidou, C., \& Yaki, K. (2016). Aerodynamic design of a MALE UAV. Aerospace Science and Technology, 50, 127-138. https://doi.org/10.1016/j. ast.2015.12.033Get

Pardessus, T. (2004). Concurrent Engineering Development and Practices for Aircraft Design at Airbus. Proceedings of the 24th ICAS Conference. http://icas.org/ICAS_ARCHIVE/ICAS2004/PAPERS/413.PDF

Parvez , A. M., \& Toppur, B. (2019). Hybrid Agile Project Management Model for New Product Development in Aerospace. International Journal of Operations and Quantitative Management.

Raymer, D. P. (2012). Aircraft Design: A Conceptual Approach. American Institute of Aeronautics and Astronautics.

Rebentisch, E., Conforto, E. C., Schuh, G., Riesener, M., Kantelberg, J., Amaral, D. C., \& Januszek, S. (2018). Agility Factors and Their Impact on Product Development Performance. International Design Conference - Design 2018, 893 -904. https://doi.org/10.21278/idc.2018.0236

Rohajawati, S., Rahayu, P., Akbar, H., Indria, S., \& Sensuse, D. (2019). Implementing DSDM and oo Method to Develop Billing in Mental Hospital. Journal of Physics: Conference Series, 1566, 26-27. https://doi.org/10.1088/1742 $-6596 / 1566 / 1 / 012059$

Roskam, J. (2004). Airplane Design. DARcorporation.

Salvato, J., \& Luplume, A. (2020). Agile Stage-Gate Management (ASGM) for physical products. $R$ and $D$ Management. https://doi.org/10.1111/radm.12426

Schuh, G. (2012). Innovation Management-HandBook Production and Management. Springer.
Schuh, G., Dolle, C., Kantelberg, J., \& Menges, A. (2018). Identification of Agile Mechanisms of Action as Basis for Agile Product Development. Procedia CIRP, 70, 19-24. https:// doi.org/10.1016/j.procir.2018.02.007

Schuha, G., Gartzena, T., Soucy-Bouchardb, S., \& Bassea, F. (2017). Enabling Agility in Product Development through an Adaptive Engineering. Procedia CIRP, 63, 342347. htps://doi.org/10.1016/j.procir.2017.03.106

Schwaber, K., \& Sutherland, J. (2016). The Scrum Guide. Scrum. org. http://www.scrumguides.org/docs/scrumguide/ v2016/2016-Scrum-Guide-US.pdf

Sohaib, O., Solanki, H., Dhaliw, N., Hussain, W., \& Asif, M. (2019). Integrating design thinking into extreme programming. Journal of Ambient Intelligence and Humanized Computing, 10, 2485-2492. doi.org/10.1007/s12652-018-0932-y

Srivastava, A., Bhardwaj, S., \& Saraswat, S. (2017). sCRUM model for agile methodology. International Conference on Computing, Communication and Automation (ICCCA), 864869. https://doi.org/10.1109/CCAA.2017.8229928

Stare, A. (2014). Agile Project Management in Product Development Projects. 27th IPMA World Congress, 295-304.

Sutherland, J. (2013). The Scrum Handbook. Scrum Inc.

Takeuchi , H., \& Nonaka, I. (1986). The New New Product Development Game. Harvard Business Review, 64(1), 137-146.

us Deparment of Defense. (2007). Unmanned Systems Roadmap 2007-2032. https://www.globalsecurity.org/inte Il/library/reports/2007/dod-unmanned-systems-road map_2007-2032.pdf

Vinodh, S., Devadasan, S. R., Maheshkumar, S., Aravindakshan, M., Arumugam, M., \& Balakrishnam, K. (2010). Agile Product Development through CAD and Rapid Prototyping Technologies: An Examination in a Traditionalan examination in a traditional pump-manufacturing company. International Journal Advance Manufacturing and Technology, 46, 663 - 679. https://doi.org/10.1007/s00170-009-2142-4 